\title{
On the history of the so-called Lense-Thirring effect
}

\author{
Herbert Pfister \\ Institut für Theoretische Physik, Universität Tübingen, Auf der Morgenstelle 14, \\ D-72076 Tübingen, Germany
}

\begin{abstract}
Some historical documents, especially the Einstein-Besso manuscript from 1913, an extensive notebook by Hans Thirring from 1917, and a correspondence between Thirring and Albert Einstein in the year 1917 reveal that most of the merit of the so-called Lense-Thirring effect of general relativity belongs to Einstein. Besides this "central story" of the effect, we comment shortly on some type of prehistory, with contributions by Ernst Mach, Benedikt and Immanuel Friedlaender, and August Föppl, and we follow the later history of the problem of a correct centrifugal force inside a rotating mass shell which was resolved only relatively recently. We also shortly comment on recent possibilities to confirm the so-called Lense-Thirring effect, and the related Schiff effect, experimentally.
\end{abstract}

Key words: Lense-Thirring effect, Dragging, Coriolis force, Centrifugal force, Mach's principle

\section{Introduction}

For Isaac Newton the experiment with the rotating bucket was the decisive reason to introduce the concept of an absolute space. In contrast, Ernst Mach argued that also this experiment may fit under the postulate of relativity of rotation if one assumes appropriate influences of (rotating) cosmic masses on local systems. At the end of the 19th century, the brothers Benedikt and Immanuel Friedlaender considered in more detail such non-Newtonian "gravitational forces", and they, and August Föppl even performed (unsuccessful) experiments to detect such forces.

Email address: herbert.pfister@uni-tuebingen.de (Herbert Pfister). 
A more systematic analysis of such additional forces began when Albert Einstein tried to generalize the Newtonian theory of gravity such that it obeys (at least locally) the principles of special relativity. On this way he first considered a scalar, relativistic gravity theory, and found therein the phenomenon of linear dragging (of test masses and inertial systems) inside a linearly accelerated mass shell. Soon afterwards he developed (with Marcel Grossmann) the tensorial Entwurf-theory, and derived now (with Michele Besso) a Coriolis type force within a rotating, spherical mass shell (which was one half of the value in final general relativity), and a motion of the nodes of planets due to the sun's rotation (which was one fourth of the value in general relativity).

Hans Thirring started in the year 1917, on the basis of general relativity, an extensive notebook "Wirkung rotierender Massen" (effect of rotating masses). The first third of this notebook considers mainly "centrifugal effects" of second order in the angular velocity $\omega$, and is of little lasting value. Only after a letter by Einstein (dated August 2, 1917) he considers also Coriolis effects (of first order in $\omega$ ), and calculates such effects near the center of a rotating mass shell, and in the far field of a rotating spherical body. Astronomical applications of these results were performed by Josef Lense. In today's literature these results run under the somewhat misleading name "Lense-Thirring effects", whereas the main merit for, and insight into this new "gravitational force" belongs to Einstein.

Thirring's notebook, and his well known publication (Thirring, 1918a) direct their main attention to a so-called centrifugal force. However, this force (as induced by rotating bodies in a laboratory, or by the rotating earth) is on one hand far below any measurability even with present technology, on the other hand it has, besides the structurally correct components, also an incorrect axial component. Also later corrections of Thirring's work by (Cornel Lanczos, 1923) and (L.Bass, \& Felix Pirani, 1955) could not cure this defect. A solution of this "centrifugal force problem" in a rotating mass shell (of mass $M$ ) requires an aspherical deformation of the shell, a flat space-time in its interior, and a treatment in orders $M^{2}$ and higher. These requirements were fulfilled not earlier than in 1985 by (Herbert Pfister, \& Karlheinz Braun, 1985). Herewith, the postulate of relativity of rotation was realized - within the model class of rotating mass shells - as completely as one can wish.

In recent years the so-called Lense-Thirring effect (of first order in $\omega$ ) has received new interest and importance because it becomes now-more than 85 years after the theoretical predictions of Einstein, Thirring, and Lense- possible to directly measure this tiny effect. (Indirect confirmations are contained in some earlier precision tests of general relativity like Lunar Laser Ranging, and the analysis of double pulsar systems.) On one hand it has been possible to follow the orbits of the geophysical LAGEOS satellites so precisely that the motion of their nodes due to the earth's rotation showed agreement with 
the predictions of general relativity within $10 \%$ (Ciufolini, \& Pavlis, 2004). On the other hand, already in the years 1959-1960 it was discovered by G. E. Pugh (1959) and Leonard Schiff (1960a,b) that the gravitomagnetic dragging phenomenon of general relativity leads to another effect-sometimes called the Schiff effect - which might be suited for experimental confirmation: The rotation axis of a gyroscope, orbiting (inside a satellite) the earth in a height of e.g. $650 \mathrm{~km}$, suffers, besides other, more dominant effects, a precession of 42 milliarcseconds per year, due to the earth's rotation. (For more details about this effect see e.g. Ignazio Ciufolini, \& John Wheeler (1995), Chap.6.) At the Hansen Laboratory of Stanford University a corresponding satellite mission, Gravity Probe B, was prepared since more than 35 years, pushing the technology to its extreme in many places (Francis Everitt et al., 2001). On April 20, 2004 the satellite was successfully launched. The period of data taking is now finished, and hopefully in 2006 the results (of predicted accuracy of $1 \%$ or better) will be communicated.

\section{The prehistory (Mach, Friedlaender, Föppl)}

Here we can be relatively short because in the book (Julian Barbour, \& Pfister, 1995) this history is extensively treated, with longer (translated) quotations from Mach, Friedlaender, and Föppl. The idea that rotating bodies may exert on test particles not only the static (Newtonian) gravitational force but an additional "dragging force" deflecting the test particles in the direction of the rotation, was presumably first formulated by Mach (1872): "Obviously it does not matter whether we think of the earth rotating around its axis, or we imagine a static earth, and the celestial bodies rotating around it." (This quotation can be seen as a type of definition for the "postulate of relativity of rotation", frequently appearing in this paper.) As is well known, Mach elaborated more in detail on these questions in his famous book on mechanics (Mach, 1883): "The principles of mechanics can, presumably, be so conceived that even for relative rotations centrifugal forces arise. Newton's experiment with the rotating vessel of water simply informs us that the relative rotation of the water with respect to the sides of the vessel produces no noticeable centrifugal forces, but that such forces are produced by its relative rotation with respect to the mass of the earth and other celestial bodies. No one is competent to say how the experiment would turn out if the sides of the vessel increased in thickness and mass till they were ultimately several leagues thick". And although Mach did not provide a concrete extension of Newton's laws of inertia and gravitation, e.g. by adding velocity-dependent forces, and although he did not perform any "dragging experiments", Mach's mechanics was a decisive stimulus for other physicists (like Friedlaender, and Föppl; see below) to do such things. Conversely, Mach reacted, in later editions of his mechanics 
(e.g. in the third edition of 1897, and in the sixth edition of 1908) quite positively to these attempts.

In 1896 the brothers Friedlaender published a very interesting booklet (Friedlaender, 1896). On one hand they formulated, in extension of Mach's work, interesting, in some cases even prophetic theoretical ideas: "It seems to me that the correct form of the law of inertia will only then have been found when relative inertia as an effect of masses on each other and gravitation, which is also an effect of masses on each other, have been derived on the basis of a unified law". At the end of the booklet, B. Friedlaender even vaguely anticipates Einstein's incorporation of inertia and gravity into the properties of space and time: "It is also readily seen that in accordance with our conception the motions of the bodies of the solar system can be regarded as pure inertial motions, whereas in accordance with the usual conception the inertial motion, or rather its gravitationally continually modified tendency, strives to produce a rectilinear tangential motion." The concrete - but of course unsuccessfulexperiment of I. Friedlaender searched for a possible influence of a rapidly rotating, heavy fly-wheel on a torsion balance mounted above the fly-wheel, in line with its axis.

A quite different, and, in principle, more promising experiment was performed by Föppl (1904). Here the rotating source was the whole earth, and the test system was a gyroscope, consisting of two heavy fly-wheels, rotating with angular velocity up to $2300 \mathrm{rpm}$. It was tested whether the rotating earth induced a Coriolis-type "dragging force" (of first order in its angular velocity $\omega$, in contrast to the $\omega^{2}$-type effects due to a centrifugal force) on the gyroscope axis, and it was found that such an effect was less than $2 \%$ of $\omega$.

\section{The central story (Einstein, Thirring, Lense)}

As is well known, Einstein started his search for a relativistic gravitation theory in (Einstein, 1907), where he introduced the equivalence principle, and derived therefrom a gravitational redshift and a light deflection. In 1912, Einstein formulated a scalar, relativistic gravitation theory (Einstein, 1912a,b), and showed that such a theory necessarily has to be nonlinear, in order to obey the equivalence principle. Within this theory, Einstein performed the first concrete calculation of a Machian dragging effect (Einstein, 1912c) ${ }^{1}$ : First he introduced the model of an infinitely thin, spherical mass shell (mass $M$, radius $R$ ), a model which is very useful until today in final general relativ-

1 The reason why this article is "hidden" in a very unusual journal is that Einstein was on very friendly terms with $\mathrm{H}$. Zangger, a professor for forensic medicine in Zürich, and the quoted volume was a birthday present to Prof. Zangger. 
ity, because (a) it represents the optimal substitute for the Newtonian mass point which is forbidden in general relativity due to the collapse phenomenon, (b) it allows to study mass effects by solving only the vacuum Einstein field equations (in the interior and exterior of the mass shell). Einstein then considered a test mass $m$ at the center of this shell and derived (within his scalar theory) that the presence of the mass shell $M$ induces an increase of $m$ by a factor $\left(1+M G / R c^{2}\right)$. On the basis of this result he calculated that if an external force exerts a linear acceleration $\Gamma$ on the mass shell, the test mass $m$ is dragged along with the acceleration $\gamma=\left(3 M G / 2 R c^{2}\right) \Gamma$. Although these results surely encouraged Einstein in his Machian point of view on the way to a consistent relativistic theory of gravity, it has to be said that the above results are obsolete from today's knowledge: It should have been doubtful already in 1912 how a mass increase due to a gravitational field should be experimentally confirmed because such a field acts universally on all physical systems and measuring instruments. Indeed, in general relativity it was, after numerous controversial claims, shown by Carl Brans (1962) that such a mass increase is only an untestable coordinate effect. Furthermore, the title of (Einstein, 1912c) is somewhat misleading because a scalar theory can hardly produce an effect analogous to the (vectorial!) electrodynamic induction.

Very soon after the paper (Einstein, 1912c), partly still in Prague but mostly after his move to Zürich in August 1912, Einstein reached decisive new findings for a relativistic gravity theory (compare (Howard, \& Stachel, 1989) for a more extensive discussion of these issues.): It should be based on a non-Euclidean (pseudo-Riemannian) geometry with metric tensor $g_{\mu \nu}$, it should be a tensorial theory with the whole energy-momentum tensor $T_{\mu \nu}$ as source of the gravitational field, and it should, if possible, be covariant with respect to general coordinate transformations. The Zürich notebook of this time (see John Norton (1984)) reveals that Einstein and the mathematician Marcel Grossmann were even considering the Ricci tensor for the "left hand side" of the field equations, and were within a hair's breadth of finding the final Einstein equations of general relativity of November 1915. But the erroneous conclusion that such a theory would not lead to the correct Newtonian limit, urged them to discard general covariance, and to propose in the so-called Entwurf-theory (Einstein, \& Grossmann, 1913) for the left hand side of the field equations a "tensor" which is covariant only with respect to a reduced class of coordinate transformations.

Whereas the Entwurf-paper contains no direct applications of the new gravitation theory, such applications were performed, immediately after finishing this paper, by Einstein with his friend Michele Besso in June 1913, in the 53 pages of the so-called Einstein-Besso manuscript. (See Klein et al. (1995), pp. 344-473, where this important manuscript is reprinted, together with extended comments.) The main objective of this manuscript was the perihelion advance of Mercury which at that time was the only observation being 
in conflict with Newton's gravity theory. However, within a weak-field approximation of the Entwurf-theory Einstein and Besso got for Mercury's perihelion advance a value of 18 arcseconds per century instead of the experimental value 43 arcseconds per century (more precisely, the value was 5/12 of the value in final general relativity). This will be the reason why Einstein never published these calculations, and it surely was one of the decisive reasons for later discarding the Entwurf-theory. (It may be remarked that Johannes Droste (1914) calculated, independently of Einstein and Besso, the same "wrong" result for the perihelion shift in the Entwurf-theory.) But besides this incorrect result for the perihelion shift, the Einstein-Besso manuscript contains some other interesting and future-pointing results: On pp. 36-37 they derive a Coriolis force inside a spherical, rotating mass shell (mass $M$, radius $R$ ), and calculate the resulting "dragging" of test particles: For the ratio $f$ between the induced angular velocity of the test particles and the angular velocity of the mass shell they get $f=4 M G / 3 R c^{2}$, half the value which Thirring derived in 1918 in final general relativity (Thirring, 1918a). This is the only part of the manuscript entering Einstein's great talk in September 1913 at the Naturforscherversammlung in Vienna (Einstein, 1913a), where he also remarks that "unfortunately the expected effect is so small that we cannot hope to verify it in terrestrial experiments or in astronomy". On p. 38 of the manuscript, Einstein and Besso derive the dragging of test particles inside a linearly accelerated mass shell: $\gamma=\left(2 M G / R c^{2}\right) \Gamma$, a factor $4 / 3$ bigger than in the scalar theory of 1912 (Einstein, 1912c), and now derived without the dubious detour of a mass increase due to a gravitational field. On pp. 45-49 of the manuscript, Einstein and Besso calculate the motion of the nodes of planets in the field of the rotating sun. If one compares their result ([eq.331] on [p.49]) with the later calculation of Lense and Thirring (1918) in final general relativity (eq.(17) on p.161), and adjusts the different notations, it is seen that the effect in the Entwurf-theory is only $1 / 4$ of the effect in general relativity. (When Einstein and Besso calculate the effect for the planets Mercury and Venus, they get, however, much too large values because they insert a wrong value for the solar mass.)

As far as we know, in the years 1915-1916 neither Einstein nor anybody else calculated any gravitomagnetic or dragging effect in the Entwurf-theory or in general relativity. In 1917 Thirring started to calculate such effects within general relativity, which, at least partly, and up to the first order in the angular velocity $\omega$, Einstein (and Besso) had already calculated with very similar methods and results in 1912-1913 in the scalar and/or the Entwurf-theory. The papers (Thirring, 1918a) and (Lense, \& Thirring, 1918) do not clearly reveal how much knowledge Thirring had of the earlier results of Einstein. But at least Einstein's speech at the 1913 Vienna congress (Einstein, 1913a) must have been known to Thirring because he gave a talk (on specific heat of crystals) at the same congress, and Einstein's speech was the main event at this congress. Furthermore, Thirring published in this time frequently in the 
same journal (Physikalische Zeitschrift, e.g. Vol.13(1912)266, Vol.14(1913)406 and 867, Vol.15(1914)127 and 180) in which Einstein's speech appeared, and in the short article on the formal analogies between the Maxwell equations and the linearized Einstein equations (Thirring, 1918b) Thirring explicitly quotes (Einstein, 1913a). Of course, Thirring did not know all details of the EinsteinBesso manuscript. However, in a letter of August 2, 1917 (see Schulmann et al. (1998)), Einstein told Thirring that he has calculated the Coriolis field of the rotating earth and sun, and its influence on the orbit elements of planets (and moons).

A later article by Thirring (Thirring, 1966), a tribute for the 50th anniversary of Mach's death, reveals that Thirring originally tried to set up an experiment for measuring centrifugal-type forces inside a heavy, rotating hollow cylinder. This is somewhat strange: Whereas Friedlaender (1896) and Föppl (1904) are excused that they did not or could not estimate the order of magnitude of the effect searched for (compare the discussion remarks by W. Rindler, J. Norton, and J. Renn in (Barbour, \& Pfister, 1995), pp. 56-57), in the year 1917, 12 years after the publication of Einstein's special relativity, it should have been clear that such a relativistic correction to Newton's gravity theory can be at most of the order $M G / R c^{2}$, i.e. below the ridiculously small value $10^{-24}$ for all conceivable laboratory systems $(M \leq 100 \mathrm{~kg}, R \geq 10 \mathrm{~cm})$. Furthermore, the quantity $M G / R c^{2}$ was explicitly contained in (Einstein, 1913a), and e.g. in the Schwarzschild solution from 1916. Thirring (1966), however, tells that he turned from the intended experiment to a theoretical calculation of the expected effect because, in the turmoil of the first world war, he could not organize the equipment for the experiment.

The history of the origin and rise of the two central papers (Thirring, 1918a) and (Lense, \& Thirring, 1918) can be disclosed quite well because the "Österreichische Zentralbibliothek für Physik" in Vienna has in storage the estate of Hans Thirring, containing a 156 pages notebook "Wirkung rotierender Massen" (Thirring, 1917). This notebook is a remarkable document, and it allows a detailed view into the workshop of a theoretical physicist of that time. In Thirring's original numbering the notebook has only 107 pages, and covers mainly the year 1917, the first explicit date, April 24, 1917, appearing on Thirring's page 17. However, the notebook contains also numerous later additions, partly on the reverse sides (pages 59R, 61R, ...), the last entry dating from July 2, 1922. In the following we try to analyze, as far as possible, Thirring's notebook in chronological order, and according to Thirring's page numbering. The pages 1-18 can be considered as a type of "warm up", with detailed calculations of metric components and Christoffel symbols, partly (e.g. pp. 8-12) crossed out by Thirring himself. On some pages Thirring speaks of the energy-momentum tensor and of the metric tensor of a rotating sphere but the relevant formulas are obviously wrong because they contain factors $\sin \omega t$ and $\cos \omega t$, being forbidden for a stationary system. The pages 15-16 
treat (again wrongly) the field of a thin ring, pages 17-18 contain calculations in connection with Einstein's cosmological paper (Einstein, 1917). On p. 19 Thirring begins a calculation of the field of a rotating spherical mass shell, first for points near the origin in the equatorial plane, from p. 23 on also for points outside this plane. But he confines himself to the diagonal components $g_{i i}$ which are of order $\omega^{2}$. On p. 24 he says (with date April 26, 1917) "the appearance of the axial force component is unintelligible to me", and then "after a discussion with Flamm I realize that the hitherto existing contradictions presumably are solved if, in the integration, the volume contraction due to the motion is taken into consideration". (From today's perspective it has to be said that the mass increase of the equatorial parts of the mass shell due to their motion is, as also discussed in (Thirring, 1918a), one reason for the appearance of the axial component of the "centrifugal force". But, as will be analyzed in more detail in Sec. 4, there are other "centrifugal effects" in a rotating mass shell, overlooked by Thirring and other authors, which in their sum allow to produce a correct centrifugal force field, without an axial component.) From p. 25 on, Thirring comes back to the rotating sphere of constant density, and he calculates the field (but again only the diagonal components $g_{\mu \mu}$ ) for points far off the sphere. The pages 32-44 treat the "field of the rotating spherical space". Presumably, Thirring has realized that the rotating sphere and the rotating mass shell with their asymptotically Minkowskian boundary conditions do not answer the Machian question concerning a static Newton bucket inside a rotating celestial sphere, and that a cosmological treatment (possibly with a cosmological constant) is necessary. (Compare the introduction to (Thirring, 1918a).) On p. 45 Thirring says: "One should publish: I. The field of the rotating sphere and of the spherical shell. II. The transformation from Einsteinto de Sitter-coordinates. III. The cosmological solution in orthogonal Einstein coordinates." Pages 49-51 contain the draft of a letter to Einstein, dated July 11 (corrected to 17), 1917, reprinted as document 361 in (Schulmann, et al., 1998). Thirring writes e.g.: "before I publish my results, I should like to send you a short report, in the hope to receive a further impulse directly from you". He tells about his calculations for the rotating mass shell and for the rotating sphere, but he gives only the $g_{44}$-components, with their contributions of order $\omega^{2}$, and he discusses these under the aspect of a centrifugal force, also mentioning the surprising axial component of the force. At the end of the letter, Thirring asks Einstein whether he could think of an experimental confirmation of such a centrifugal effect on the innermost moon of Jupiter. Einstein's answer from August 2, 1917 (document 369 in (Schulmann, et al., 1998)) is quite short, but it exposes the weak points in Thirring's work (up to this time) in an admirably clear and concise way: "To your example of the hollow sphere it is only to be added that, besides the centrifugal field whose axial component you interpret so nicely, also a Coriolis field results which corresponds to the components $g_{41}, g_{42}, g_{43}$ of the potential, and which is proportional to the first power of $\omega$. This field acts orthogonally deflecting on moving masses, and produces e.g. a rotation of the pendulum plane in the Foucault experiment. I 
have calculated this dragging for the earth; it stays far below any measurable amount. Such a Coriolis field is produced also by the rotation of the sun and of Jupiter, and it causes secular changes of the orbital elements of the planets (respectively the moons) which, however, stay far below the measurement error. ... Nevertheless, the Coriolis fields seem to be accessible to measurement more easily than your correction terms to $g_{44}$ because the latter have the same symmetry properties as the field distortion due to oblateness."

The first entries in Thirring's notebook after the receipt of Einstein's letter (pages 54 and 55, with dates September 5 and 11, 1917) deal with topics he has never considered before: "Calculation of $g_{14}, g_{24}$, and $g_{34}$ for the rotating spherical shell", and "Determination of the Coriolis force from $g_{24}$ ". The notebook then has a time-gap of 2 months, where Thirring had to do practical work, presumably for the military authorities, as Thirring's second letter to Einstein from December 3, 1917 (document 401 in (Schulmann, et al., 1998)) indicates. The pages 56-83, covering the period November 14-29, 1917, take up the calculations for the rotating shell and the rotating sphere, but now adding to the centrifugal force type terms of order $\omega^{2}$ also the Coriolis force type terms of order $\omega$, requested by Einstein. Interspersed are here comments on a comparison with the Maxwell equations (later published in (Thirring, $1918 \mathrm{~b})$ ), and on the field of a uniformly moving mass point. The pages 84-92, dating from November 29 to December 14, 1917, contain a first draft (mostly in shorthand) of the article (Thirring, 1918a), but here under the title "On the question of the relativity of rotational motions in Einstein's gravity theory". As already mentioned, on December 3, 1917 Thirring writes a second letter to Einstein, whose draft is also contained in the notebook. He tells that he prepares two articles (Thirring, 1918a) and (Lense, \& Thirring, 1918) for publication. He then addresses a problem, also appearing at the end of (Thirring, 1918a): By transforming the interior of the rotating mass shell to a coordinate system rotating with an appropriate angular velocity $\omega^{\prime}$, one can eliminate the Coriolis force. But, according to Thirring's results, the centrifugal force does not vanish in the same rotating system. Thirring mentions also some pretended problem with the energy balance. In his immediate answer of December 7, 1917 (document 405 in (Schulmann, et al., 1998)) Einstein, on one hand, reveals the error leading to the pretended violation of the energy balance. Einstein also tries to answer Thirring's first question on the "relativity of rotation", but here he is not really successful, especially when he says that this is already guaranteed by the general covariance of the equations of the theory. The pages 93-99 of Thirring's notebook, covering the period December $7-15,1917$, mainly contain further calculations on the rotating mass shell and on the rotating sphere, and considerations about the reference system rotating with angular velocity $\omega^{\prime}$. The final paper (Thirring, 1918a) was then received by the publishers on December 21, 1917. (There exists also a paper (Thirring, 1918c) - the write-up of a lecture of November 6, 1917 at the chemical-physical society of Vienna-which summarizes the contents of (Thirring, 1918a) but 
has a more general and qualitative character. In the estate of Hans Thirring at the "Österreichische Zentralbibliothek für Physik" in Vienna there exists also a typescript (Thirring, 1922) which coincides partly with (Thirring, 1918c) but is even more qualitative.)

The pages 101-105, dated January 25-28, 1918, contain the draft (again mostly in shorthand) of $\S \S 1-2$ of the paper (Lense, \& Thirring, 1918). Here, Thirring omits the partly very involved expressions of order $\omega^{2}$, worked out in the notebook, and confines himself to the terms of first order in $\omega$. Since Thirring's notebook contains no details of $\S 3$ : "Calculation of the perturbations due to the proper rotation of the central body" (transformation of the equations of motion in Cartesian coordinates in $\S 2$ to the orbital elements used in astronomy), and of $\S 4$ : "Numerical results" of the paper (Lense, \& Thirring, 1918), it is plausible that these (and only these) parts were calculated and formulated by J. Lense. This supposition is supported by the sentence "My collegue, Prof. Lense, ... has then taken over the task to compare the results with observations on stars" in Thirring's later article (Thirring, 1966), and by the short article (Lense, 1918). The paper (Lense, \& Thirring, 1918) was received by the publishers on February 21, 1918. As already remarked earlier, the calculations in (Lense, \& Thirring, 1918) are only performed for the far field, i.e. for distances $r$ from the center of the rotating body (radius $R$ ) with $r / R \gg 1$. Therefore, the calculations as such apply neither to the Gravity Probe B experiment (Everitt, et al., 2001) with $r / R \approx 1.10$, nor to the measurements with the LAGEOS satellites (Ciufolini, \& Pavlis, 2004) with $r / R \approx 1.92$. However, a continuation of the calculations of (Lense, \& Thirring, 1918) to higher orders of $R / r$ reveals that for the exterior gravitational field of a slowly rotating, spherical body all these higher order terms vanish, and that the Coriolis acceleration $\vec{b}$ for a test mass of velocity $\vec{v}$ reads for all values $r / R>1$

$$
\vec{b}=2 \vec{v} \times \vec{H}, \quad \text { with } \quad \vec{H}=\frac{2 M G R^{2}}{5 c^{2} r^{3}}\left[\vec{\omega}-3 \frac{(\vec{\omega} \vec{r}) \vec{r}}{r^{2}}\right] .
$$

(More elegantly, it is already clear from symmetry considerations that a first order rotational perturbation of a spherical system can only produce a pure dipole field proportional to $r^{-3}$.)

For completeness, we add some remarks on the rest of Thirring's notebook: pages 106-107, and some additional (unnumbered) pages contain the draft and a reprint of the paper (Thirring, 1918b); on the reverse of pages 21 and 24, and on pages 59R, 61R, 62R, 95R, and 96R Thirring treats (in the period October 11-14, 1920) the "correction after Pauli" (an error in the integration volume), which was then published as an erratum in Physikalische Zeitschrift 22 (1921), pp. 29-30; pages 63R-72R (from the period June 30 July 2, 1922) contain a "correction after Jaffé", and a "letter to Jaffé" (in 
short hand); this is a reaction to the paper (Jaffé, 1922) that compares different formulas for the "mass change" in a gravitational field, and criticizes Thirring's formula at the end of the paper (Thirring, 1918a). As already mentioned in the beginning of Sec. 3, according to (Brans, 1962) all such "mass changes" are pure coordinate effects in general relativity, and therefore are obsolete from today's perspective.

From our analysis in this section we come to the following conclusion concerning the respective merits of Einstein, Thirring, and Lense for the so-called Lense-Thirring effect: Einstein surely had the first idea that such a dragging effect should result from a relativistic, tensorial theory of gravity. He introduced the extremely useful model of a rotating mass shell into the game, and he calculated (in the Entwurf-theory) the Coriolis force field inside a rotating shell (Einstein, 1913a), and the motion of the nodes of planets due to the sun's rotation (Klein, et al., 1995). Herein he achieves with a minimum of calculational expense (of only first order in the angular velocity $\omega$ ) a maximum of physical insight and practical results. Since the calculated effects were, however, below measurability at that time, and because Einstein presumably realized that the effects in final general relativity will not differ qualitatively from his results in the Entwurf-theory, he did not repeat the calculations after November 1915. But it was Einstein who, through his letter of August 2, 1917, brought Thirring on the track of the dragging effects (of first order in $\omega$ ) in general relativity which today run under the label "Lense-Thirring effects". Moreover, Lense and Thirring did not realize that their result (1) is also valid in the range $1<r / R<2$, and is therefore applicable to "low-orbit" satellites like Gravity Probe B and LAGEOS. Thirring's notebook (Thirring, 1917) surely is a document of high calculational power and endurance, but it is somewhat deficient in physical insight, and in a sense for reality or measurability. (In Thirring's own memoirs he admits that "in contrast to my fruitfulness as a teacher, my achievements as researcher are rather meagre although not devoid of interest". See (Zimmel, \& Kerber, 1992), p.21.) Possibly due to the fact that Mach's discussion of Newton's bucket is centered on centrifugal effects, and that also in daily life centrifugal effects usually dominate over Coriolis effects, Thirring is so fixed to these centrifugal effects that, until Einstein's intervention, he never considers the order $\omega$-Coriolis terms. Obviously, Thirring never estimated the order of magnitude of the calculated centrifugal effects, which are far below measurability under realistic circumstances, even with today's technology. Furthermore, Thirring missed many centrifugal effects being active in a rotating mass shell, and therefore never got a correct centrifugal force field. (See Sec. 4 for more details.) Nevertheless, even Thirring's published article (Thirring, 1918a) puts more weight on the centrifugal effects than on the Coriolis effects, notwithstanding Einstein's request to the contrary. And even if one bears in mind that in the time of Einstein's and Thirring's papers the standards of quoting references, and acknowledging contributions by colleagues, have been different from today's practice, it appears as very strange 
that in the papers (Thirring, 1918a) and (Thirring, \& Lense, 1918) neither the paper (Einstein, 1913a) is quoted, nor the decisive stimuli by Einstein's letter are acknowledged. As already mentioned, the contributions of Lense obviously consist (only) of the astronomical evaluations of Thirring's formulas. Therefore, we argue that the so-called Lense-Thirring effect should, historically more correct and fair, be called the Einstein-Thirring-Lense effect. But we are aware that there will be no chance to correct the designation, which has already entered textbooks, after so long time.

\section{The aftermath (The problem of a correct centrifugal force field)}

The essential results for the acceleration field $\vec{a}$ inside a slowly rotating, spherical mass shell in (Thirring, 1918a) have been

$$
\vec{a}=-2 d_{1}(\vec{\omega} \times \vec{v})-d_{2}[\vec{\omega} \times(\vec{\omega} \times \vec{r})+2(\vec{\omega} \vec{r}) \vec{\omega}],
$$

with $d_{1}=4 M G / 3 R c^{2}$, and $d_{2}=4 M G / 15 R c^{2}$ (after the Pauli correction). However, as already mentioned in Sec. 3, Thirring confined his calculations to points $\vec{r}$ near the origin, i.e. with $r / R \ll 1$. He did not realize that the first (Coriolis-)term in (2) is valid for all points with $r / R<1$, according to the same symmetry arguments mentioned in connection with the Lense-Thirring field (1) outside a rotating spherical body. In contrast, the second ("centrifugal") term in (2) would receive contributions from higher order terms in $r / R$, but, as already indicated, the derivation of this term anyhow suffers from many physical deficiencies which were realized and corrected only very gradually, some of them not earlier than 67 years after Thirring's paper.

The first essential deficiency of Thirring's result (2) was found by Lanczos (1923): Thirring explicitly says that he disregards any stresses in the shell material, and he starts from a dust-like energy-momentum tensor $T^{\mu \nu}$. But this energy-momentum tensor does (in the space-time calculated by Thirring) not fulfil the "conservation law" $T^{\mu \nu}{ }_{i}=0$, so that the calculated gravity field does not really solve Einstein's field equations everywhere. In order that the mass elements of the shell can rotate on spherical orbits, the centrifugal forces have to be compensated by appropriate stresses in the shell material. This has, according to Lanczos, the consequence that the spatial components $T^{i k}$ of the energy-momentum tensor have to vanish, and that the factor $d_{2}$ in equation (2), again only calculated for $r \ll R$, is reduced to half its value. But the problem with the axial component $2(\vec{\omega} \vec{r}) \vec{\omega}$ of the "centrifugal force" persists. More than three decades later, Bass and Pirani (1955) partly repeat Lanczos' arguments but present them in more mathematical detail, and generalize Thirring's model to a latitude-dependent mass density $\rho(\theta)=\rho_{o}\left(1+N \frac{\omega^{2} R^{2}}{c^{2}} \sin ^{2} \theta\right)$ of the 
shell, with constants $\rho_{o}$ and $N$. (Obviously independently, Hönl and Maue (1956) derive at the same time similar but less complete results.) Bass and Pirani call the choice $N=-1$ "the most interesting case": "This corresponds to a mass distribution which just compensates for the special-relativistic increase in density, and represents a uniform mass distribution in the reference frame in which the shell is rotating. This choice leads to the annihilation of both the radial and the axial 'centrifugal' forces, leaving the 'Coriolis' force intact." In the introduction of (Bass, \& Pirani, 1955) it is noticed that in Thirring's model the self-interaction of the shell (being proportional to $M^{2}$ ) is neglected, but it is not tried to push the model beyond the weak field approximation of first order in $M$. Another argument, calling for a treatment of the rotating mass shell at least up to order $M^{2}$, was presented by Charlotte Soergel-Fabricius (1960, 1961): As already discussed in (Thirring, 1918a), it is possible to eliminate the Coriolis acceleration inside the rotating mass shell by a transformation to an appropriately rotating reference system. However, the centrifugal acceleration can vanish in the same reference system, as it should according to Mach's demand for relativity of rotation, at best if it is of order $\left(M G \omega / R c^{2}\right)^{2} r$, instead of the order $\left(M G \omega / R c^{2}\right) \omega r$ in (Thirring, 1918a).

A treatment of the rotating mass shell even exactly in $M$ was then started by Brill and Cohen (1966), by considering a rotational perturbation not of Minkowski spacetime but of the Schwarzschild solution. However, they confined themselves to the first order in the angular velocity $\omega$, and derived (for the whole, flat interior of the shell) a Coriolis-type acceleration, with dragging factor (compare equation (2))

$$
d_{1}=\frac{4 \alpha(2-\alpha)}{(1+\alpha)(3-\alpha)}
$$

with $\alpha=M G / 2 R c^{2}$, and where $R$ denotes the shell radius in isotropic coordinates. (In Schwarzschild coordinates the expression would be somewhat more involved.) In the weak field limit $M G \ll R c^{2}$, this dragging factor coincides of course with Thirring's result $d_{1}=4 M G / 3 R c^{2}$. But the central new result of Brill and Cohen is that in the collapse limit $R \rightarrow M G / 2 c^{2}$ the dragging factor attains the value $d_{1}=1$. This signifies - within the model class of rotating mass shells, and up to first order in $\omega-$ a complete realization of the Machian postulate of relativity of rotation: In the collapse limit the interior of the shell ties off (as a type of separate universe) from the exterior space-time, and interior test bodies and inertial frames are dragged along with the full angular velocity $\omega$ of the shell. It has, however, to be admitted that near the collapse limit the shell material attains somewhat unphysical properties: For $R<3 M G / 4 c^{2}$, the dominant energy condition (see Hawking, \& Ellis, 1973) is violated, and in the final collapse limit the stresses in the shell diverge. 
An extension of the Brill and Cohen results to higher orders in $\omega$, and especially the long-standing problem of the induction of a correct centrifugal force by rotating masses, had to wait for another 19 years for a solution (Pfister, \& Braun, 1985). The solution is based on two "new" observations which could and should have been made already in Thirring's time, but which, for unexplicable reasons, were overlooked by all authors before 1985:

a) Any physically realistic, rotating body will suffer a centrifugal deformation in orders $\omega^{2}$ and higher, and cannot be expected to keep its spherical shape.

b) If we aim and expect to realize in the interior of the rotating mass shell quasi-Newtonian conditions with "correct" Coriolis and centrifugal forcesand no other forces! - , the interior of the mass shell obviously has to be a flat piece of space-time. In first order of $\omega$, this flatness is more or less trivial because the only non-Minkowskian metric component $g_{t \phi}$ is constant there, i.e. we have a constantly rotating Minkowski metric, and therefore a structurally correct Coriolis force. In contrast, in order $\omega^{2}$ this flatness is by no means trivial, and it is indeed violated for Thirring's solution, due to the axial component of his "centrifugal force". Moreover, if Thirring would have extended his calculations to orders $\omega^{3}, \omega^{4}, \ldots$ he would have obtained additional forces in the interior of the rotating mass shell, in conflict with Newtonian physics in a rotating reference system.

With these observations, the problem of a correct centrifugal force inside a rotating mass shell boils down to the question whether it is possible to connect a flat interior metric through a mass shell (with, to begin with, unknown geometrical and material properties) to the non-flat but asymptotically flat exterior metric of a rotating body. In full generality, this would represent a mathematically quite intricate free boundary value problem for the stationary and axisymmetric Einstein equations. However, if we confine ourselves to a perturbation expansion in the angular velocity $\omega$, all metric functions can be expanded in spherical harmonics, respectively, due to the axial symmetry, just in Legendre polynomials $P_{l}(\cos \theta)$, where in order $\omega^{n}$ the index $l$ is limited by $l \leq n$. In this way, the Einstein equations reduce to a system of ordinary differential equations for the functions $f_{l}^{(i)}(r)$ multiplying $P_{l}(\cos \theta)(i=1, \ldots, 4$, for the 4 different metric coefficients describing the stationary, axisymmetric space-time in the exterior of the mass shell).

According to (Pfister, \& Braun, 1985), in order $\omega^{2}$ the shell geometry is given by $r_{S}=R\left(1+\omega^{2} c_{2} P_{2}(\cos \theta)\right)$, with a constant $c_{2}$, and with corresponding corrections in higher (even) orders $\omega^{2 n}$. Furthermore, it turns out (Pfister, \& Braun, 1986) that in order $\omega^{3}$ the flatness of the interior space-time can only be maintained if the shell material rotates differentially, $\omega_{S}=\omega\left(1+\omega^{2} e_{2} P_{2}(\cos \theta)\right)$, with a constant $e_{2}$, and with corresponding corrections in higher (odd) orders $\omega^{2 n+1}$. Surprisingly, the flatness condition enforces a prolate form of the shell: invariant equatorial circumference smaller than the invariant polar circumference. The conditions that the exterior metric (written 
e.g. in the isotropic coordinate $r$ ) is asymptotically flat, and joins, at $r=r_{S}$, continuously to the interior (rotating) flat metric, lead (for given $M$ and $R$ ) to a unique determination of the constants $c_{2 n}$ and $e_{2 n}$, and of the functions $f_{l}^{(i)}(r)$. The energy-momentum tensor $T_{\mu \nu}$ of the shell material results then uniquely from the discontinuities of the radial derivatives of $f_{l}^{(i)}(r)$ at $r=r_{S}$, and $T_{\mu \nu}$ is $\theta$-dependent in orders $\omega^{2}$ and higher. Only in the collapse limit, the rotating shell with flat interior is spherical, and rigidly rotating, and it produces the Kerr geometry in the exterior, as was already deduced by de la Cruz and Israel (1968). For a mass shell which deviates from sphericity already in zeroth order of $\omega$, there is no solution with flat interior (Pfister, 1989).

Finally, we should like to comment on the cosmological relevance of the work of Einstein, Thirring, and later authors, concerning dragging of inertial frames. It is clear that Mach envisaged a realization of his ideas about relativity of rotation, if any, only in a cosmological context. Therefore, the work of Einstein, Thirring, and others, which confirmed some aspects of this 'relativity of rotation' in the model class of rotating mass shells, was often criticized for the asymptotic flatness of the exterior solution, instead of using cosmological boundary conditions. And Thirring himself seems to have felt an uneasiness about this 'defect' when on pages 32-44 of his notebook (Thirring, 1917) he considered the cosmological "field of the rotating spherical space". From today's knowledge it can be said that the essential dragging results for rotating bodies and mass shells in an asymptotically flat background carry over with only minor changes to cosmological boundary conditions: As shown by Christian Klein (1993), it is possible to embed a slowly rotating mass shell with flat interior also in a (rotationally perturbed) Friedmann universe, and the resulting dragging factor compares reasonably with the results of (Thirring, 1918a) and (Brill, \& Cohen, 1966), but depends of course also on the type of Friedmann cosmos $(k=0, \pm 1)$, and on its mass density.

\section{References}

Barbour,J., \& Pfister,H. (Eds.) (1995). Mach's Principle: From Newton's Bucket to Quantum Gravity. Boston: Birkhäuser.

Bass,L., \& Pirani,F.A.E. (1955). On the Gravitational Effects of Distant Rotating Masses. Philosophical Magazine, 46, 850-856.

Brans,C.H. (1962). Mach's Principle and the Locally Measured Gravitational Constant in General Relativity. Physical Review, 125, 388-396.

Brill,D., \& Cohen,J.M. (1966) Rotating Masses and their Effect on Inertial Frames. Physical Review, 143, 1011-1015.

Ciufolini,I., \& Pavlis,E.C. (2004). A confirmation of the general relativistic prediction of the Lense-Thirring effect. Nature, 431, 958-960. 
Ciufolini,I, \& Wheeler,J.A. (1995). Gravitation and Inertia. Princeton: Princeton University Press.

de la Cruz,V., \& Israel,W. (1968). Spinning Shell as a Source of the Kerr Metric. Physical Review, 170, 1187-1192.

Droste,J. (1914). On the Field of a Single Centre in Einstein's Theory of Gravitation. Koninkle Akademie van Wetenschapen te Amsterdam, Proceedings of the Section of Sciences, 17, 998-1011.

Einstein,A. (1907). Über das Relativitätsprinzip und die aus demselben gezogenen Folgerungen. Jahrbuch der Radioaktivität und Elektronik, 4, 411-462 (Chap.V, pp. 454-462).

Einstein,A. (1912a). Lichtgeschwindigkeit und Statik des Gravitationsfeldes. Annalen der Physik, 38, 355-369.

Einstein,A. (1912b). Zur Theorie des statischen Gravitationsfeldes. Annalen der Physik, 38, 443-458.

Einstein,A. (1912c). Gibt es eine Gravitationswirkung, die der elektrodynamischen Induktionswirkung analog ist? Vierteljahrschrift für gerichtliche Medizin und öffentliches Sanitätswesen, 44, 37-40.

Einstein,A. (1913). Zum gegenwärtigen Stande des Gravitationsproblems. Physikalische Zeitschrift, 14, 1249-1266.

Einstein.A. (1917). Kosmologische Betrachtungen zur allgemeinen Relativitätstheorie.

Sitzungsberichte der Königlich Preussischen Akademie der Wissenschaften, Berlin, 142-152.

Einstein,A., \& Grossmann,M. (1913). Entwurf einer verallgemeinerten Relativitätstheorie und einer Theorie der Gravitation. Leipzig und Berlin: Teubner. Everitt,C.W.F., et al. (2001). Gravity Probe B: Countdown to Launch. Gyros, Clocks, Interferometers, ... : Testing Relativistic Gravity in Space. Springer Lecture Notes in Physics 562, pp. 52-82. Berlin: Springer.

Föppl,A. (1904). Über einen Kreiselversuch zur Messung der Umdrehungsgeschwindigkeit der Erde. Sitzungsberichte der Bayerischen Akademie der Wissenschaften, 34, 5-28. (Summary in (Barbour, \& Pfister, 1995).)

Friedlaender,B., \& I. (1896). Absolute oder relative Bewegung? Berlin: Verlag Simion. (Partial translation, and comments in (Barbour, \& Pfister, 1995).)

Hönl,H., \& Maue,A.W. (1956). Über das Gravitationsfeld rotierender Massen. Zeitschrift für Physik, 144, 152-167.

Howard,D. \& Stachel,J. (Eds.) (1989). Einstein and the History of General Relativity. Boston: Birkhäuser.

Jaffé,G. (1922). Ruhmasse und Masse der Bewegung im statischen Gravitationsfelde. Physikalische Zeitschrift, 23, 337-340.

Klein,C. (1993). Rotational perturbations and frame dragging in a Friedmann universe. Classical and Quantum Gravity, 10, 1619-1631.

Klein,M.J., et al. (Eds.) (1995). The Collected Papers of Albert Einstein, Vol. 4. Princeton: Princeton University Press.

Lanczos,K. (1923). Zum Rotationsproblem der allgemeinen Relativitätstheorie. Zeitschrift für Physik, 14, 204-219.

Lense,J. (1918) Über Relativitätseinflüsse in den Mondsystemen. Astronomis- 
che Nachrichten, 206, 117-120.

Lense,J., \& Thirring,H. (1918). Über den Einfluß der Eigenrotation der Zentralkörper auf die Bewegung der Planeten und Monde nach der Einsteinschen Gravitationstheorie. Physikalische Zeitschrift, 19, 156-163. (Translation in General Relativity and Gravitation 16 (1984), pp. 727-741.)

Mach,E. (1872). Die Geschichte und die Wurzel des Satzes von der Erhaltung der Arbeit. Prag: Calve'sche Buchhandlung.

Mach,E. (1883). Die Mechanik in ihrer Entwicklung. Historisch-kritisch dargestellt. Leipzig: F.A. Brockhaus.

Norton,J. (1984). How Einstein found his Field Equations, 1912-1915. Historical Studies in the Physical Sciences, 14, 253-316.

Pfister,H. (1989). Rotating mass shells with flat interiors. Classical and Quantum Gravity, 6, 487-503.

Pfister,H., \& Braun,K.H. (1985). Induction of correct centrifugal force in a rotating mass shell. Classical and Quantum Gravity, 2, 909-918.

Pfister,H., \& Braun,K.H. (1986). A mass shell with flat interior cannot rotate rigidly. Classical and Quantum Gravity, 3, 335-345.

Pugh,G.E. (1959). Proposal for a satellite test of the Coriolis prediction of general relativity. WSEG Research Memorandum No.11. Washington, DC: The Pentagon.

Schiff,L.I. (1960a). Possible new experimental test of general relativity theory. Physical Review Letters, 4, 215-217.

Schiff,L.I. (1960b). Motion of a gyroscope according to Einstein's theory of gravitation. Proceedings of the National Academy of Sciences of the USA, 46, 871-882.

Schulmann,R., et al. (Eds.) (1998). The Collected Papers of Albert Einstein, Vol.8. (Documents 361, 369, 401, and 405) Princeton: Princeton University Press.

Soergel-Fabricius,C. (1960). Thirring-Effekt im Einsteinkosmos. Zeitschrift für Physik, 159, 541-553.

Soergel-Fabricius,C. (1961). Über den Ursprung von Coriolis- und

Zentrifugalkräften in stationären Räumen. Zeitschrift für Physik, 161, 392-403.

Thirring,H. (1917). Wirkung rotierender Massen. Notebook in the Österreichische Zentralbibliothek für Physik, Universität Wien.

Thirring,H. (1918a). Über die Wirkung rotierender ferner Massen in der Einsteinschen Gravitationstheorie. Physikalische Zeitschrift, 19, 33-39. (Translation in General Relativity and Gravitation 16 (1984) pp. 712-725.)

Thirring,H. (1918b). Über die formale Analogie zwischen den elektromagnetischen Grundgleichungen und den Einsteinschen Gravitationsgleichungen erster Näherung. Physikalische Zeitschrift, 19, 204-205.

Thirring,H. (1918c). Über die Relativität der Rotationsbewegung in der Einsteinschen Gravitationstheorie. Vierteljahresberichte des Wiener Vereins zur Förderung des physikalischen und chemischen Unterrichts, 23, 17-29. 
Thirring,H. (1922). Über die Relativität der Rotationsbewegung in der Einsteinschen Gravitationstheorie. Typescript in the Österreichische Zentralbibliothek für Physik, Universität Wien.

Thirring,H. (1966). Ernst Mach als Physiker. Almanach der Österreichischen Akademie der Wissenschaften, 166, 361-372.

Zimmel,B., \&Kerber,G. (Eds.) (1992). Hans Thirring. Ein Leben für Physik und Frieden. Wien: Böhlau. 